\title{
CAPÍTULO 35: PERFIL SENSORIAL E AVALIAÇÃO FÍSICA DE COOKIES COM FARINHA MISTA EXTRUDADA DE CASCAS E ALBEDO DE MARACUJÁ E ARROZ
}

\section{CHAPTER 35: SENSORY PROFILE AND PHISICAL EVALUATION OF COOKIES WITH EXTRUDED MIXED FLOUR OF PEEL AND PASSION FRUIT ALBEDO AND RICE}

Valéria França de Souza ${ }^{1}$; Nandara Gabriela Mendonça Oliveira ${ }^{2}$; Rafael Henrique de Almeida Ferreira ${ }^{3}$, Alyne Alves Nunes Oliveira ${ }^{4}$, José Luís Ramirez Ascheri ${ }^{5}$

\begin{abstract}
Resumo
Os co-produtos (cascas e albedo) de maracujá é um resíduo que pode ser aproveitado na forma de farinha. $\mathrm{O}$ arroz apresenta característica de hipoalergenicidade, com flavor suave e isento de glúten. Este trabalho teve como objetivo desenvolver formulações de biscoitos tipo cookie sem glúten contendo uma farinha mista extrudada de cascas e albedo de maracujá(Passiflora edulis flavicarpa Degener) e arroz (Oryza sativa L.) e avaliação física. Para os cookies avaliou-se a aceitabilidade sensorial e avaliação física. A análise sensorial do cookies foi realizada com 100 julgadores não treinados, utilizando uma escala hedônica de 9 pontos, mediante a assinatura do Termo de Consentimento Livre e Esclarecido. Para determinar a avaliação física do cookies foi realizado valores médios da massa, volume aparente, volume específico, diâmetro, espessura e fator de expansão. Os resultados obtidos mostram que das três formulações (Fb8, Fb12 e Fb13) desenvolvidas, duas (Fb8 e Fb13) obtiveram aceitação por parte dos julgadores. Os resultados da avaliação física indicaram que o diâmetro e o fator de expansão obtiveram os maiores valores. Portanto, concluí-se que o uso de co-produtos (cascas e albedo de maracujá) na fabricação de farinha para cookies pode ser uma alternativa viável para o consumo por portadores de doença celíaca.
\end{abstract}

Palavras-Chaves: fruta, cereal, extrusão termoplástica, biscoito, free glúten.

\begin{abstract}
Passion fruit co-products (peels and albedo) is a residue that can be used in the form of flour. Rice is hypoallergenic, with a mild flavor and free of gluten. This work aimed to develop gluten-free cookie type formulations containing a mixed flour extruded from peel and passion fruit albedo (Passiflora edulis flavicarpa Degener) and rice (Oryza sativa L.) and physical evaluation. Sensory acceptability and physical evaluation were evaluated for cookies. Sensory analysis of cookies was performed with 100 untrained judges, using a hedonic scale of 9 points, by signing the Informed Consent Form. To determine the physical evaluation of cookies, average values of mass, apparent volume, specific volume, diameter, thickness and expansion factor were performed. The results obtained show that of the three formulations (Fb8, Fb12 and Fb13) developed, two (Fb8 and Fb13) obtained acceptance by the judges. The results of the physical evaluation indicated that the diameter and the expansion factor obtained

\footnotetext{
${ }^{1}$ Doutora do Curso de Pós Graduação em Ciência e Tecnologia de Alimentos, Universidade Federal Rural do Rio de Janeiro, vssouzafrana@gmail.com

${ }^{2}$ Graduanda em Engenharia de Alimentos, Universidade Federal Rural do Rio de Janeiro, nandaragabriela@yahoo.com.br

${ }^{3}$ Mestrando do Curso de Mestrado em Ciência e Tecnologia em Alimentos, Instituto Federal do Sudeste de Minas Gerais, rafaelhenrique93@gmail.com

${ }^{4}$ Doutoranda do Curso de Pós Graduação em Higiene Veterinária e Processamento Tecnológico de Produtos de Origem Animal , Universidade Federal Fluminense do Rio de Janeiro, alves.alyne@ hotmail.com

${ }^{5}$ Pesquisador da Embrapa Agroindústria de Alimentos, Rio de Janeiro, joseascheri@gmail.com
} 
the highest values. Therefore, it was concluded that the use of co-products (peels and passion fruit albedo) in the manufacture of flour for cookies can be a viable alternative for consumption by people with celiac disease.

Keywords: fruit, cereals, thermosplastic extrusion, biscuit, free glúten.

\section{Introdução}

O Brasil é o maior produtor mundial de maracujá (IBGE, 2018), sendo que $60 \%$ desta produção é destinada ao consumo in natura e o restante destinado a industrialização (MELETTI, 2011). De acordo com dados fornecidos pelo Instituto Brasileiro de Geografia e Estatística (IBGE, 2018) o Brasil colheu em 2018 uma área de aproximadamente 42.731 hectares, com uma produção por volta de 602.651 toneladas, destacando a região nordeste como a maior produtora. O maracujá também pode ser considerado uma rica fonte de carotenóides, pigmentos responsáveis pela cor amarela intensa de seu suco. Esses nutrientes, juntamente com flavonóides, também presentes, possuem caráter antioxidante, atuando na prevenção de doenças crônicas(ZERAIK et al., 2010). Vale destacar que devido ao seu "flavour" único e delicado, os frutos do maracujá têm sido assunto de diversas pesquisas, resultando na caracterização de inúmeros constituintes voláteis. Nesse sentido mais de 40 substâncias já foram identificadas, com ésteres, aldeídos, cetonas e alcoóis, responsáveis pelo aroma desta fruta (ZERAIK, 2010). Zucolotto et al., (2012) identificaram várias substâncias bioativas nos frutos, folhas e cascas presentes em várias espécies de Passiflora edulis, tendo como componente principal os flavonóides C-glicosídeos.

Além disso, alguns estudos apontam para o potencial benéfico da casca do maracujá é composta pelo flavedo (parte com coloração) e albedo (parte branca), sendo este rico em pectina, espécie de fibra solúvel que auxilia na redução das taxas de glicose no sangue, fonte de vitamina B3, ferro, cálcio e fósforo (CÓRDOVA et al., 2005). Do ponto de vista tecnológico, Yapo e Koffi (2006) destacam que a casca de maracujá amarelo é, em especial, uma boa fonte de pectina de baixa metoxilação. Zeraik e Yariwake (2012), ao avaliar extratos de cascas de maracujá azedo (amarelo), observaram alta capacidade antioxidante. As propriedades funcionais da casca do maracujá, especialmente aquelas relacionadas ao teor e o tipo de fibras, fazem com que a casca de maracujá não seja mais considerada um resíduo industrial, uma vez que pode ser utilizada na elaboração de novos produtos na forma de farinha (GALISTEO, DUARTE, ZARZUELO, 2008). Segundo Zeraik ; Yariwake (2012), as cascas podem ser utilizadas para produção de vários alimentos, contribuindo assim para reduzir grandes quantidades de resíduos orgânicos gerados no processamento de suco de fruta. 
Para Abud e Narain (2009), as cascas de maracujá devem ser considerada como farinha de fibras residual do maracujá amarelo, devido às suas características e propriedades funcionais, tornando-as de grande potencial econômico e nutricional.

O arroz é o alimento básico mais importante para a nutrição humana, amplamente consumido, e capaz de fornecer energia e nutrientes para mais da metade da população mundial (PANG et al., 2018). Além de não ser alergênica, a farinha de arroz é um produto versátil, tem sabor suave, baixos níveis de sódio e alta proporção de amido facilmente digerível (NABESHIMA e EL-DASH,2004; SIVARAMAKRISHNAN et al.,2004; OLIVEIRA et al., 2014). O uso da farinha de arroz permite melhorar a qualidade nutricional dos produtos por extrusão, como também apresentar pequenos tamanhos dos grânulos de amido, alta proporção de amidos facilmente digeríveis e ampla faixa de teor de amilose (AWOLU et al., 2015).

A extrusão termoplástica de alimentos é definida como um processo termomecânico pelo qual, materiais amiláceos e/ou proteáceos são plastificados com água e cozidos em um tubo, pela combinação de câmbios de pressão, transferência de calor e massa e cisalhamento mecânico (BERK, 2009).

O Brasil tem importante posição como exportador de biscoitos, sendo que os principais mercados, em 2017, foram Angola, Venezuela, Cuba, Argentina, Estados Unidos (EUA), Uruguai, Paraguai, Peru, Chile e Japão (ABIMAPI,2018).

Segundo BRAM et al.(2009) e GÖKMEN et al. (2008), os “cookies” são definidos como produtos assados à base de cereais que possuem altos níveis de açúcar e de gordura e baixos níveis de água (1-5\%). Os biscoitos tipo cookie, tem as características peculiares, possuem crocância referente a massa que leva menos líquido se comparado com outros biscoitos e a modulagem (GISSLEN, 2014).

Atualmente, no mercado nacional e internacional, é possível encontrar biscoitos isentos de glúten, porém, muitos produtos disponíveis no mercado não apresentam boa aceitação pelos consumidores (ZUCCO et al., 2011). Os biscoitos tipo cookie podem ser reformulados por farinhas isentas de glúten, tornando-se assim uma opção para pessoas que possuem alergia e/ou intolerância à proteína gliadina, contida no glúten (RAHAIE et al., 2014).

A doença celíaca é caracterizada como uma desordem sistêmica autoimune, desencadeada pela incapacidade de digestão da proteína conhecida como glúten (principal fração proteica presente nos cereais trigo, centeio, triticale, cevada) em indivíduos geneticamente predispostos (FENACELBRA, 2010). Na presença de doença celíaca, os 
alimentos que contêm glúten devem ser substituídos por outros, como por exemplo: milho, arroz, soja, batata e mandioca, de forma que a dieta atenda às necessidades nutricionais de acordo com a idade do indivíduo (FARO 2008; LA BARCA et al., 2010). Apesar de sua crescente oferta, os produtos livres de glúten nem sempre têm boa qualidade sensorial e possuem custo elevado. Daí a importância da pesquisa e desenvolvimento de novos produtos que se enquadrem nessa categoria (FERREIRA et al., 2009).

Este trabalho teve como objetivo desenvolver formulações de biscoitos tipo cookie sem glúten contendo uma farinha mista extrudada de cascas e albedo de maracujá(Passiflora edulis flavicarpa Degener) e arroz (Oryza sativa L.) e avaliação física.

\section{Material e Métodos}

\section{Obtenção das matérias primas}

\section{Arroz}

O arroz (Oryza sativa L.) sob a forma de grãos polidos, da marca Tio João, classe longo fino tipo 1 , em embalagem de $5 \mathrm{Kg}$ e mantidas à temperatura ambiente até o processo de moagem.

\section{Maracujá}

O maracujá (Passiflora Edulis Flavicarpa Degener) para a extração da casca (epicarpo) e do albedo (mesocarpo) foram adquiridos no comércio local da cidade do Rio de Janeiro.

Os experimentos foram desenvolvidos na planta-piloto de cereais da Embrapa Agroindústria de Alimentos, localizado em Guaratiba, Rio de Janeiro, RJ.

\section{Obtenção da farinha de arroz}

$\mathrm{O}$ arroz foi retirado das embalagens em que são comercializados e moídos em moinho de disco, com peneira de abertura de $1 \mathrm{~mm}$, obtendo-se então a farinha de arroz branco.

\section{Obtenção da farinha de cascas e albedo de maracujá}

O preparo da farinha de maracujá (casca + albedo) foi realizado em frutas in natura. As frutas foram selecionadas, lavadas em água corrente, para retirada de sujeiras e sanitizadas com água clorada (20ppm) de cloro residual livre durante 20 minutos). Em seguida foram lavadas novamente em água corrente para a retirada do cloro residual. Foi feita a divisão do maracujá em 4 partes (despolpamento), em seguida foi retirada a polpa e, a casca e o albedo foram secos em estufa a $70^{\circ} \mathrm{C}$ durante 4 horas. Após a secagem, as cascas e albedo foram pesados e acondicionados em sacos plásticos para serem posteriormente moídos. As amostras secas foram moídas em moinho de facas-martelo Marca TREU com peneira de $1 \mathrm{~mm}$, em seguida 
moinho de disco com abertura de $2 \mathrm{~mm}$ e posterior o moinho de perten com peneira de $0,8 \mathrm{~mm}$ obtendo-se a farinha da casca e albedo de maracujá com granulometria adequada. A determinação da distribuição granulométrica realizada em triplicata da farinha de (casca+albedo) foi determinada em um analisador e tamanho de partícula a laser Analysette 22 (Fritsch, Idar-Oberstein, Alemanha). Os experimentos foram realizados em triplicata. A farinha de casca e albedo de fruto foi acondicionada em sacos plásticos, identificadas e mantidas armazenadas a temperatura de refrigeração de $4^{\circ} \mathrm{C}$ para posterior uso.

\section{Delineamento Experimental}

Foi utilizado o delineamento experimental composto central (Tabelas 1 e 2 ) visando reduzir as combinações e otimizar o processo de coleta e análise de dados, segundo BOX et al (1987). Foram analisados as variáveis independentes, temperatura na terceira zona do canhão; formulação da farinha de arroz e farinha de casca e de albedo de maracujá e umidade de processamento.

Tabela 1. Níveis das variáveis independentes do processo de elaboração dos extrudados expandidos.

\begin{tabular}{llllll}
\hline Variáveis & $-\alpha=1,682$ & -1 & 0 & +1 & $+\alpha=1,682$ \\
$\mathrm{X}^{1}$ & 99,6 & 120 & 150 & 180 & 200,4 \\
$\mathrm{X}^{2}$ & 14,64 & 16 & 18 & 20 & 21,36 \\
$\mathrm{X}^{3}$ & 1,6 & 5 & 10 & 15 & 18,4 \\
\hline $\mathrm{X}^{1}$ - Temperatura das zonas para extrusora Brabender $\left({ }^{\circ} \mathrm{C}\right)$ &
\end{tabular}

$\mathrm{X}^{2}$ - Umidade de processamento $(\%)$

$\mathrm{X}^{3}$ - Formulação da farinha da casca e albedo de maracujá (\%); a diferença da percentagem corresponde à farinha de arroz 
Tabela 2. Delineamento completo do desenho experimental.

\begin{tabular}{|c|c|c|c|c|c|c|}
\hline \multirow[t]{2}{*}{ Experimento } & \multicolumn{6}{|c|}{ Níveis Codificados das Variáveis Níveis Decodificados das Variáveis } \\
\hline & $\mathrm{x}^{1}$ & $x^{2}$ & $\mathrm{x}^{3}$ & $\mathrm{X}^{1}$ & $\mathrm{X}^{2}$ & $\mathrm{X}^{3}$ \\
\hline 01 & +1 & +1 & +1 & 180 & 20 & 15 \\
\hline 02 & -1 & +1 & +1 & 120 & 20 & 15 \\
\hline 03 & +1 & -1 & +1 & 180 & 16 & 15 \\
\hline 04 & -1 & -1 & +1 & 120 & 16 & 15 \\
\hline 05 & +1 & +1 & -1 & 180 & 20 & 5 \\
\hline 06 & -1 & +1 & -1 & 120 & 20 & 5 \\
\hline 07 & +1 & -1 & -1 & 180 & 16 & 5 \\
\hline 08 & -1 & -1 & -1 & 120 & 16 & 5 \\
\hline 09 & $+1,68$ & 0 & 0 & 200,4 & 18 & 10 \\
\hline 10 & $-1,68$ & 0 & 0 & 99,6 & 18 & 10 \\
\hline 11 & 0 & $+1,68$ & 0 & 150 & 21,36 & 10 \\
\hline 12 & 0 & $-1,68$ & 0 & 150 & 14,64 & 10 \\
\hline 13 & 0 & 0 & $+1,68$ & 150 & 18 & 18,4 \\
\hline 14 & 0 & 0 & $-1,68$ & 150 & 18 & 1,6 \\
\hline 15 & 0 & 0 & 0 & 150 & 18 & 10 \\
\hline 16 & 0 & 0 & 0 & 150 & 18 & 10 \\
\hline 17 & 0 & 0 & 0 & 150 & 18 & 10 \\
\hline 18 & 0 & 0 & 0 & 150 & 18 & 10 \\
\hline 19 & 0 & 0 & 0 & 150 & 18 & 10 \\
\hline 20 & 0 & 0 & 0 & 150 & 18 & 10 \\
\hline
\end{tabular}

$\mathrm{x}^{1}, \mathrm{x}^{2}$ e $\mathrm{x}^{3}=$ níveis codificados

$\mathrm{X}^{1}=$ Temperatura das zonas para extrusora Brabender $\left({ }^{\circ} \mathrm{C}\right)$

$\mathrm{X}^{2}=$ Umidade de processamento $(\%)$

$\mathrm{X}^{3}=$ Formulação da farinha da casca e albedo e maracujá (\%)

Utilizou-se neste estudo três diferentes combinações dos melhores tratamentos $T_{8}, T_{12}$ e $\mathrm{T}_{13}$. Os experimentos das variáveis foram (farinha de casca e albedo de maracujá + arroz) $\mathrm{T}_{8}:$ 5:95; $\mathrm{T}_{12}: 10: 90$ e $\mathrm{T}_{13}: 18,4: 81,6$; umidade de processamento $\mathrm{T}_{8}: 16 \%, \mathrm{~T}_{12}: 14,64 \%$ e $\mathrm{T}_{13}$ : $18 \%$ e a temperatura na $3^{\mathrm{a}}$ zona da extrusora do $\mathrm{T}_{8}: 120^{\circ} \mathrm{C}, \mathrm{T}_{12}$ e $\mathrm{T}_{13}: 150^{\circ} \mathrm{C}$.

Utilizou-se o delineamento em metodologia de superfície de resposta do tipo central composto rotacional de $2^{\mathrm{a}}$ ordem em testes preliminares para 20 tratamentos. As variáveis independentes foram codificados $(-\alpha,-1,0,+1,+\alpha)$ de acordo com a matriz circular de $3 \mathrm{~mm}$, temperatura na $1^{\mathrm{a}}$ zona $60^{\circ} \mathrm{C}$; e temperatura na $2^{\mathrm{a}}$ zona $100^{\circ} \mathrm{C}$. As temperaturas na $1^{\mathrm{a}}$ e $2^{\mathrm{a}}$ zona foram fixas no experimento. Os parâmetros variáveis foram controle da temperatura na $3^{\mathrm{a}}$ zona de aquecimento do canhão da extrusora. Previamente a etapa de preparação da farinha mista, as amostras formuladas em diferentes percentagens de farinha de arroz e farinha de casca e albedo de maracujá foram homogeneizadas por 5 minutos e acondicionadas para os diferentes níveis de umidade e formulação (ASCHERI e CARVALHO, 2015). A farinha mista foi ajustada a umidade inicial em triplicata de acordo com o delineamento experimental. 
As amostras formuladas da farinha mista foram acondicionadas para os diferentes níveis de umidade e formulação.

Os teores de umidade inicial das farinhas mistas foram determinados por gravimetria através da dessecação do material a $105^{\circ} \mathrm{C}$ até peso constante em estufa (INSTITUTO ADOLFO LUTZ, 1985).

Após a determinação da umidade das farinhas mistas, a equação (1) foi usada para estabelecer a quantidade de água a ser adicionada às amostras, a fim de se alcançar os níveis de umidade estabelecidos para cada formulação.

$$
\mathrm{Y}=\left[\frac{(\mathrm{Uf}-\mathrm{Ui}) \times \mathrm{Pa}}{100-\mathrm{Uf}}\right]
$$

Onde:

$\mathbf{Y}=$ quantidade de água a ser adicionada $(\mathrm{mL})$

$\mathbf{U f}=$ umidade final (\%) (base seca)

$\mathbf{U i}=$ umidade inicial $(\%)$ (base seca)

$\mathbf{P a}=$ peso da amostra $(\mathrm{g})$

\section{Análise Estatística}

O processamento dos dados e a análise estatística foram realizados com auxílio do programa computacional Statistica Six Sigma, versão 7.0, com as variáveis independentes codificadas.

\section{Condições do Processo de Extrusão}

Para a elaboração dos extrudados foi utilizado uma extrusora monorosca, marca Brabender, modelo DSE 20 DN, (Duisburg, Alemanha), com sistema de extrusão através de fricção mecânica, taxa de compressão do parafuso (3:1), taxa de alimentação: $2,5 \mathrm{Kg} / \mathrm{h}$, sistema de refrigeração pneumático, para controle de temperatura na camisa de extrusão, velocidade de rotação do parafuso a $140 \mathrm{rpm}$, capacidade de produção aproximada de $16 \mathrm{Kg} . \mathrm{h}^{-1}$ e matriz de saída circular de $3 \mathrm{~mm}$ de espessura.

\section{Processo de Extrusão Termoplástica}

As farinhas mistas de casca e albedo de maracujá e arroz foram processadas em uma extrusora monorosca.

O início da extrusão do material formulado foi realizado após as três zonas do parafuso (alimentação, transição e alta pressão) alcançarem as temperaturas pré estabelecidas. A 
temperatura da $1^{\mathrm{a}}$ zona foi mantida em $60^{\circ} \mathrm{C}$, a temperatura da $2^{\mathrm{a}}$ zona a $100^{\circ} \mathrm{C}$ e a temperatura da $3^{\mathrm{a}}$ zona variou conforme o delineamento experimental (Tabela 2).

Os extrudados expandidos após a saída da matriz foram secos em um secador com circulação de ar forçado a $70^{\circ} \mathrm{C}$ por 4 horas ou até que a umidade final seja inferior a $4 \%$ (base seca).

\section{Desenvolvimento das formulações}

A Tabela 3 contém as formulações dos biscoitos tipo cookie sem glúten elaborados com farinha de extrudados mista de cascas e albedo de maracujá e arroz.

Tabela 3. Formulações desenvolvidas para elaboração de biscoitos do tipo cookie sem glúten à base de farinha de extrudados mista de cascas e albedo de maracujá e arroz com substituição parcial de 5\%,10\% e 18,4\%.

\begin{tabular}{lccc}
\hline \multicolumn{4}{c}{ Tipos de Formulações de Farinha de cascas e albedo de maracujá } \\
\hline Ingredientes & $\begin{array}{c}5 \% \\
\left(\mathrm{Fb}_{8}\right)^{*}\end{array}$ & $\begin{array}{c}10 \% \\
\left(\mathrm{Fb}_{12}\right)^{*}\end{array}$ & $\begin{array}{c}18,4 \% \\
\left(\mathrm{Fb}_{13}\right)^{*}\end{array}$ \\
\hline Amido de milho (g) & 160 & 120 & 80 \\
Farinha extrudada (casca e albedo & 40 & 80 & 120 \\
de maracujá e arroz (g) & & & \\
Açúcar refinado (g) & 80 & 80 & 80 \\
Gordura de palma (g) & 60 & 60 & 60 \\
Bicarbonato de sódio (g) & 2 & 2 & 2 \\
Sal refinado (g) & 1 & 1 & 1 \\
Lecitina de soja (ml) & 2 & 2 & 2 \\
Baunilha (ml) & 2 & 2 & 4 \\
Soro de Leite (g) & 4 & 4 & 2 \\
Gema de ovo (ud) & 2 & 2 & 2 \\
Bicarbonato de amônia(g) & 2 & 2 & \\
\hline
\end{tabular}

NOTA: Porcentagens dos ingredientes calculadas em relação ao peso do amido de milho.

* valores correspondentes a \% de farinha da casca e albedo de maracujá; Fb: Formulação do biscoito

Fonte: Valéria F. de Souza

\section{Processamento de biscoitos}

Para a obtenção do biscoito tipo cookies com as farinhas mistas de extrudados de cascas e albedo de maracujá e arroz, foram obtidas a partir de um delineamento inteiramente casualizado, com três tratamentos de misturas para cookies. $\mathrm{O}_{8}$ com $5 \%$ de farinha de cascas e albedo de maracujá (FCAM) e 95\% de farinha de arroz (FA) e o $\mathrm{T}_{12}$ com $10 \%$ de FCAM e $90 \%$ de FA e o $\mathrm{T}_{13}$ com $18,4 \%$ de FCAM e $81,6 \%$ de FA, respectivamente. Para o processamento dos biscoitos tipo cookies composta por amido de milho ( $\mathrm{T}_{8}: 160 \mathrm{~g}$, $\left.\mathrm{T}_{12}: 120 \mathrm{~g}, \mathrm{~T}_{13}: 80 \mathrm{~g}\right)$, farinha extrudada de cascas e albedo de maracujá e arroz $\left(\mathrm{T}_{8}: 40 \mathrm{~g}, \mathrm{~T}_{12}: 80 \mathrm{~g}\right.$, $\mathrm{T}_{13}: 80 \mathrm{~g}$ ) e demais ingredientes fixos para os $\mathrm{T}_{8}, \mathrm{~T}_{12}$ e $\mathrm{T}_{13}$ com adição de açúcar refinado $(80 \mathrm{~g})$, gordura de palma $(60 \mathrm{~g})$, bicarbonato de sódio $(2 \mathrm{~g})$, sal refinado (1g), lecitina de soja $(2 \mathrm{ml})$, baunilha $(2 \mathrm{ml})$, soro de leite $(4 \mathrm{~g})$, gema de ovo(2 uds) e bicarbonato de amônia $(2 \mathrm{~g})$. 
Todos os ingredientes foram pesados separadamente em uma balança digital e as misturas para cookies foram obtidas em duas fases, onde se elaborou primeiro um creme com: lecitina de soja, açúcar refinado, gordura de palma, soro de leite, sal e ovos). Essa, fase onde se produziu o creme, foi efetuada por 3 minutos e na segunda fase fez-se a incorporação das farinhas (farinha extrudada de cascas e albedo de maracujá e arroz, amido de milho, bicarbonato de amônia, baunilha e fermento) ao creme para homogeneização na batedeira planetária (Arno Deluxe SX80, Brasil) por 5 minutos para a obtenção da massa. Após o processo de mistura, é realizada a abertura da massa laminar a $(2 \mathrm{~cm})$ sendo então estendida com o auxílio de um rolo em uma superfície de mármore e moldar com forma retangular obtendo-se a modelagem do biscoito. Após essa etapa foi realizada o corte da massa. Em seguida, os biscoitos foram assados em fôrma previamente forrada com papel manteiga, o forneamento ocorreu a $220^{\circ} \mathrm{C}$, por 10 minutos, em forno elétrico industrial pré-aquecido (Marca Suggar). Após o forneamento os biscoitos foram resfriados à temperatura ambiente. Após uma hora de resfriamento, foram realizadas as análises físicas dos cookies, com exceção da força de quebra, que foi realizada posteriormente, com biscoitos armazenados em temperatura ambiente e colocados em recipientes fechados hermeticamente. Após o resfriamento foram acondicionados em embalagens plásticas hermeticamente fechados.

\section{Avaliação Física dos Produtos Panificáveis}

A avaliação física foi realizada em três biscoitos em um único experimento, sendo determinados os valores médios da massa, volume aparente, volume específico, diâmetro, espessura e fator de expansão.

\section{Rendimento}

Os produtos panificáveis antes e após o forneamento foram pesados, e o rendimento (R) foi determinado através da fórmula abaixo.

$$
R=\frac{\text { Peso antes do forneament }(g)}{\text { Peso após } o \text { forneamenb }(g)}
$$

\subsubsection{Diâmetro e espessura}

O diâmetro dos biscoitos após o forneamento foi determinado com régua de escala milimetrada, e a espessura com paquímetro expressa em milímetros, após o forneamento de acordo com os procedimentos descritos no método 10-50D da INSTITUTO ADOLFO LUTZ, 1985. 


\section{Fator de expansão}

O fator de expansão (FE) foi determinado pela razão entre os valores de diâmetro e espessura dos biscoitos após o forneamento (AACC, 1995).

$$
F E=\frac{\text { Diâmetrodo produto }(\mathrm{mm})}{\text { Espessurado produto }(\mathrm{mm})}
$$

\section{Volume específico}

O volume específico (VE) dos produtos elaborados foi calculado pela relação entre o volume aparente (determinado pelo método de deslocamento da massa ocupada (de sementes de painço), e o peso dos biscoitos após o forneamento (determinando o seu volume em proveta graduada) (EL-DASH, CAMARGO e DIAZ, 1982). A determinação do volume específico foi realizado com 10 repetições. O cálculo será feito como abaixo disposto.

$$
V E=\frac{\text { Volume do produto }\left(\mathrm{cm}^{3}\right)}{\text { Peso do produto }(\mathrm{g})}
$$

\section{Análise sensorial dos biscoitos}

Esse estudo foi submetido e aprovado pelo Comitê de Ética na Pesquisa da Universidade Federal Rural do Rio de Janeiro sob o protocolo de $n^{\circ} 379 / 2013$ e os julgadores que participaram da análise sensorial assinaram um Termo de Consentimento Livre e Esclarecido elaborado com a Resolução n¹96/96 do Conselho Nacional de Saúde.

Os biscoitos foram avaliados sensorialmente no laboratório de Análise Sensorial na Embrapa Agroindústria de Alimentos, para os atributos de aparência, aroma, sabor, textura e aceitação. Foi constituída por julgadores da Instituição da Empresa (Embrapa Agroindústria de Alimentos localizado em Guaratiba-RJ) e Universidade Federal Rural do Rio de Janeiro (UFRRJ), incluindo estudantes e funcionários vinculado a ela.

O painel foi constituído por 100 julgadores não treinados, de ambos os sexos e faixas etárias, os quais avaliaram os biscoitos tipo cookie sem glúten com farinha mista extrudada de cascas e albedo de maracujá e arroz, respectivamente pela escala hedônica de aceitação. Segundo IFT (1981), cita que os julgadores não necessitam de treinamento, porém, são selecionados para representar uma população alvo. 
Cada julgador recebeu três amostras, com os diferentes tratamentos, pesando aproximadamente variando entre 5,56 a 9,22g cada, distribuídas em blocos completos casualizados de forma aleatória em pratos descartáveis e identificados com códigos de três dígitos.

Foi fornecido aos julgadores água mineral à temperatura ambiente para limpeza das papilas gustativas e uma ficha de avaliação sensorial, a qual continha uma escala estruturada de 9 pontos, ancorada pelos eixos:1, correspondente "desgostei extremamente, e 9, a gostei extremamente (STONE e SIDEL, 1985).

\section{Resultados e Discussão}

\section{Avaliação Física dos Produtos Panificáveis}

$\mathrm{Na}$ Tabela 4 estão os resultados da avaliação física do biscoito tipo cookie com farinha de cascas e albedo de maracujá e arroz.

Tabela 4. Características físicas dos biscoitos tipo cookies contendo cascas e albedo de maracujá

\begin{tabular}{|c|c|c|c|c|c|c|c|}
\hline Formulações & MAF(g) & $\operatorname{MDF}(\mathrm{g})$ & DDF(cm) & $\mathbf{E}(\mathbf{c m})$ & $\mathbf{F E}$ & $\mathbf{V A}(\mathbf{m L})$ & VE(mL.g $\left.{ }^{-1}\right)$ \\
\hline $\begin{array}{l}5 \% \text { de farinha } \\
\text { de cascas e } \\
\text { albedo de } \\
\text { maracujá }\end{array}$ & $\begin{array}{c}6,924 \pm \\
0,731516^{\mathrm{a}}\end{array}$ & $\begin{array}{c}5,701 \pm \\
0,625805^{\mathrm{a}}\end{array}$ & $\begin{array}{c}5,3349 \pm \\
0,142165^{a}\end{array}$ & $\begin{array}{c}0,685 \pm \\
0,112492^{\mathrm{a}}\end{array}$ & $\begin{array}{c}7,9963 \pm \\
1,421624^{\mathrm{a}}\end{array}$ & $\begin{array}{c}84,5 \pm \\
5,502525^{a}\end{array}$ & $\begin{array}{c}14,9132 \pm \\
1,17469796^{a}\end{array}$ \\
\hline $\begin{array}{lr}10 \% & \text { de } \\
\text { farinha } & \text { de } \\
\text { cascas } & \mathrm{e} \\
\text { albedo } & \text { de } \\
\text { maracujá } & \end{array}$ & $\begin{array}{c}6,806 \pm \\
0,590503^{b}\end{array}$ & $\begin{array}{c}5,794 \pm \\
0,52865^{\text {a }}\end{array}$ & $\begin{array}{c}4,9846 \pm \\
0,296036^{a}\end{array}$ & $\begin{array}{c}0,6726 \pm \\
0,10488^{a}\end{array}$ & $\begin{array}{c}7,621 \pm \\
1,612821^{\mathrm{a}}\end{array}$ & $\begin{array}{c}86,5 \pm \\
2,415229^{a}\end{array}$ & $\begin{array}{c}15,054 \pm \\
1,4978062^{\mathrm{a}}\end{array}$ \\
\hline $\begin{array}{lr}18,4 \quad \% & \text { de } \\
\text { farinha } & \text { de } \\
\text { cascas } & \mathrm{e} \\
\text { albedo } & \text { de } \\
\text { maracujá } & \end{array}$ & $\begin{array}{c}7,845 \pm \\
0,622276^{c}\end{array}$ & $\begin{array}{c}6,827 \pm \\
0,538476^{b}\end{array}$ & $\begin{array}{c}5,0229 \pm \\
0,122078^{a}\end{array}$ & $\begin{array}{c}0,6034 \pm \\
0,078952^{\mathrm{a}}\end{array}$ & $\begin{array}{c}8,4435 \pm \\
1,035903^{b}\end{array}$ & $\begin{array}{c}79,5 \pm \\
1,581139^{a}\end{array}$ & $\begin{array}{c}11,7001 \pm \\
0,80205354^{a}\end{array}$ \\
\hline
\end{tabular}

Letras diferentes na mesma coluna possuem diferença significativa pelo teste de Tukey $(\mathrm{p}<0,05)$. MAF: massa antes forneamento; MDF: massa depois do forneamento; DDF: diâmetro depois do forneamento; E: espessura; FE: fator de expansão (DDF/E); VA: volume aparente; VE: volume específico(VA/MDF).F $5 \%$ de farinha de cascas e albedo de maracujá; $F_{12}$ : Formulação com $10 \%$ de farinha de cascas e albedo de maracujá e $\mathrm{F}_{13}$ : Formulação com 18,4\% de farinha de cascas e albedo de maracujá.

Fonte: Valéria F. de Souza 
As características físicas dos cookies (Tabela 4), mostra que não houve aumento no diâmetro após o forneamento de todas as amostras, não havendo diferença entre as formulações (F8, F12 e F13) dos cookies.

O diâmetro dos cookies (Tabela 4) apresentou maiores valores entre as melhores formulações $\left(\mathrm{F}_{8}, \mathrm{~F}_{12}\right.$ e $\left.\mathrm{F}_{13}\right)$ comparado aos citados por Oliveira e Curta (2014) identificaram o diâmetro para os cookies elaborados com farinha de banana verde foi de 3,9. Por outro lado, Silva, Pinto e Soares (2018) identificaram o aumento no diâmetro do cookie depois do forneamento foi de 6,15 resultado superior a $[518$ presente estudo.

Verifica-se que o fator de expansão dos cookies apresentou maiores valores com substituição parcial de farinha de cascas e albedo de maracujá entre as formulações $\left(\mathrm{F}_{8}-5 \%\right.$, $\mathrm{F}_{12}-10 \%$ e $\left.\mathrm{F}_{13}-18,4 \%\right)$. Assim, pelo presente estudo verifica-se que o fator de expansão apresentou resultados maiores que Feddern et al. (2011) para fibra do farelo de arroz e farelo de trigo (contendo 10\%, 20\% e 30\%) adicionada na massa que reduziu a expansão dos biscoitos. De acordo com Santos, Storck e Fogaça (2014) os valores de fator de expansão dos biscoitos de farinha de casca de limão variaram de 5,9 a 6,4. Por outro lado, Silva, Pinto e Soares (2018) identificaram o fator de expansão para cookie enriquecido de farinha de amêndoa de pequi foi de 0,42 .

No estudo de Mariani et al.(2015), os biscoitos sem glúten, elaborados a partir de farinha de arroz, farelo de arroz e farinha de soja apresentaram menor fator de expansão (4,45\% com farinha de arroz e farinha de soja), (4,77\% com farelo de arroz e farinha de soja) e $(5,09 \%$ com farinha de arroz, farelo de arroz e farinha de soja). No presente estudo, observou-se um aumento na expansão dos cookies quando se aumentou o teor de farinha de cascas e albedo de maracujá conforme a Tabela 4.

Os cookies contendo $5 \%$ de cascas e albedo de maracujá apresentaram maior espessura. Por outro lado, Feddern et al. (2011) relatou que os biscoitos elaborados na proporção de 10 e $30 \%$ de farelo de arroz apresentaram maior espessura. As fibras das formulações $\mathrm{F}_{12}$ e $\mathrm{F}_{13}$ contendo $10 \%$ e 18,4\% de cascas e albedo de maracujá apresentaram menor espessura. Para Mariani et al.(2015), a espessura de biscoito tipo cookie elaborados com farinha de arroz, farelo de arroz e farinha de soja apresentaram menor espessura comparados ao presente estudo. A espessura dos cookies elaborados com farinha de cascas e albedo de maracujá e arroz após o forneamento não diferiram estatisticamente entre si ( $p>0,05)$. Comparando os resultados obtidos com Oliveira e Curta (2014) que desenvolveram cookie isento de glúten obtido com biomassa e farinha de banana (Musa paradisíaca) verde verificaram que após o forneamento a espessura foi de 0,8 . Verifica-se que a espessura dos 
cookies da Tabela 4 apresentou valores menores em relação ao citado por Oliveira e Curta (2014). Tal fato pode ser explicado pelos ingredientes e isentos de quantidade de água elaborados para os cookies, mesmo utilizando percentuais diferenciados de farinha de cascas e albedo de maracujá e arroz entre as formulações.

O volume específico dos cookies na Tabela 4 não afetou estatisticamente.

As massas dos cookies antes e depois do forneamento apresentaram diferença significativa entre si para as formulações na Tabela 4.

Não houve diferença significativa $(p>0,05)$ entre as formulações de cookies, quanto ao volume aparente conforme a Tabela 4.

\section{Aceitabilidade dos biscoitos tipo cookies}

A Tabela 5 mostram o índice de aceitação com relação aos diferentes atributos avaliados para os biscoitos tipo cookie sem glúten à base de arroz e cascas e albedo de maracujá com as distintas formulações.

Tabela 5. Médias e desvio padrão do teste de aceitação sensorial dos biscoitos tipo cookies utilizando concentrações de farinha de cascas e albedo de maracujá adicionada a farinha de arroz.

\begin{tabular}{|c|c|c|c|c|c|c|c|c|c|c|c|c|}
\hline & \multicolumn{4}{|c|}{$\begin{array}{c}\text { Fb8 } \\
5 \% * \\
\end{array}$} & \multicolumn{3}{|c|}{$\begin{array}{c}\mathbf{F b}_{12} \\
10 \% *\end{array}$} & & \multicolumn{4}{|c|}{$\begin{array}{c}\mathbf{F b}_{13} \\
18,4 \%\end{array}$} \\
\hline Aparência & 6,26 & \pm & 1,91 & b & 5,82 & \pm & 1,97 & b & 6,98 & \pm & 1,50 & a \\
\hline Aroma & 6,91 & \pm & 1,44 & a & 5,91 & \pm & 1,74 & b & 6,75 & \pm & 1,45 & a \\
\hline Sabor & 6,70 & \pm & 1,55 & a & 5,41 & \pm & 2,11 & b & 6,63 & \pm & 1,62 & a \\
\hline Textura & 6,90 & \pm & 1,50 & a & 4,72 & \pm & 1,95 & b & 7,06 & \pm & 1,49 & a \\
\hline Aceitação & 6,68 & \pm & 1,50 & a & 5,26 & \pm & 1,95 & b & 6,75 & \pm & 1,49 & a \\
\hline
\end{tabular}

Letras diferentes em cada linha significa que as amostras foram significativamente diferentes $(\mathrm{p}<0,05)$

*Porcentagem da farinha de cascas e albedo de maracujá incorporada à farinha de arroz.

Fonte: Valéria F. de Souza

Adicionalmente, no estudo de Nascimento et al. (2020) sobre a elaboração de biscoito com a farinha da casca do maracujá (Passiflora edulis), nas proporções de $10 \%$ e $20 \%$ da farinha, com a nota entre 8 e 7 (gostei muito e gostei moderadamente) obteve melhor aceitabilidade em relação a aceitação global comparado ao presente estudo. Ainda, os biscoitos com maior índice de aceitabilidade foram os com $5 \%$ e $18,4 \%$ e os com menor índice de aceitabilidade foi o com $10 \%$ da farinha da casca e albedo de maracujá.

Quanto ao atributo aparência, as formulações diferiram estatisticamente entre si $(\mathrm{p}<0,05)$. Esses resultados apresentados da elaboração de biscoito com a farinha da casca do maracujá (Passiflora edulis) citado por Nascimento et al. (2020) podem ser considerados superiores 
comparados as formulações com farinha de casca e albedo de maracujá em relação ao atributo aparência. Ramos et al. (2018) reportaram médias de aceitabilidade para aparência superiores $(8,0$ e 7,1$)$ ao desta pesquisa.

A Tabela 5 mostra que a diferença de aroma das formulações diferiram estatisticamente entre si $(\mathrm{p}<0,05)$. Esses índices são muito inferiores (Tabela 5) se comparados aos biscoitos elaborados com 10\%, 20\% e 30\% nas formulações de farinha da casca do maracujá (Passiflora edulis) desenvolvidos por Nascimento et al. (2020). Já para os autores Ramos et al. (2018) relataram a aceitabilidade de biscoitos tipo cookie enriquecidos com farinha de jatobá e afirmaram que os biscoitos adicionados de $10 \%$ e $20 \%$ de farinha de jatobá a sua formulação não diferiram estatisticamente entre si $(\mathrm{p}<0,05)$ em relação ao aroma.

Com relação ao sabor, foi demonstrado diferença estatística significativa $(p<0,05)$ entre as formulações. Para as Fb8 e Fb13 apresentaram semelhanças neste atributo. Por outro lado, os biscoitos com farinha da casca do maracujá (Passiflora edulis) elaborados por Nascimento et al.(2020) apresentaram para o atributo sabor as formulações $\mathrm{A}(8,30)$ e $\mathrm{B}(7,42)$ maiores aceitabilidade. Ramos et al.(2018) ao avaliarem sensorialmente biscoitos tipo cookie com farinha de jatobá, obtiveram médias superiores ao deste trabalho para o atributo sabor.

Quanto à textura, a melhor formulação foi a Fb13. Nascimento et al. (2020) elaboraram três formulações de biscoitos com farinha da casca do maracujá, e obtiveram no atributo textura valor maior de aceitação nas formulações $A(7,85)$ e $B(7,69)$. Assim pelo presente estudo verifica-se que os resultados apresentados para textura de biscoito com farinha da casca e albedo de maracujá e arroz foram inferiores quando comparado as formulações A e B, de biscoitos processados com farinha da casca do maracujá (Passiflora edulis) citado por Nascimento et al. (2020). Por outro lado, Ramos et al. (2018) estudou a aceitabilidade dos biscoitos tipo cookie enriquecidos com farinha de jatobá e mencionaram que para o atributo crocância os biscoitos não apresentaram diferença significativa nas formulações elaboradas com $10 \%$ e $20 \%$ de farinha de jatobá. Neste estudo encontramos valores superiores quanto à textura para cookies formulados com 18,4\% de farinha de cascas e albedo de maracujá e arroz em comparação aos encontrados por Oliveira et al. (2017) nas formulações B $(6,94)$ e C $(6,44)$.

\section{Conclusões}

Os biscoitos cookie isento de glúten enriquecidos com farinha mista de cascas e albedo de maracujá e arroz apresentaram aumento de diâmetro e do fator de expansão. 
Sensorialmente, os biscoitos mais aceitáveis quanto à aparência, textura e aceitação foram os biscoitos com 18,4\% da Fb13, sendo aqueles com 5\% da Fb8 considerados com melhor aroma e sabor.

A aceitabilidade da Fb13 foi satisfatória, indicando que o cookie desenvolvido apresenta potencial para inclusão em produtos isentos de glúten.

A incorporação de $18,4 \%$ de farinha de cascas e albedo de maracujá em biscoitos, verifica-se que os biscoitos mais apreciados constaram daqueles com maior percentual de fibras.

Em relação aos biscoitos contendo $10 \%$ de farinha de cascas e albedo de maracujá foram considerados os menos aceitáveis quanto aos atributos sensoriais.

Portanto, concluí-se que o uso de co-produtos (cascas e albedo de maracujá) na fabricação de farinha para cookies pode ser uma alternativa viável para o consumo por portadores de doença celíaca.

\section{Referências}

ABIMAPI. Associação Brasileira das Indústrias de Biscoitos, Massas. Disponível em http://www.investimentosenoticias.com.br/noticias/negocios/abimapi-registra-crescimento-de50-das-exportacoes-em-2017. Acesso em outubro de 2018.

ABUD, A.K.S.; NARAIN, N. Incorporação da farinha de resíduo do processamento de polpa de fruta em biscoitos: uma alternativa de combate ao desperdício. Brazilian Journal of Food Technology, v.12, n.4, p.257-265, 2009.

AMERICAN ASSOCIATION OF CEREAL CHEMISTS (AACC). Approved methods of American Association of Cereal Chemists. $9^{\text {th }}$ ed. St. Paul, 1995.

ASCHERI, J. L. R.; CARVALHO, C.W.P. Apostila do curso anual em processo de extrusão de alimentos: aspectos tecnológicos para o desenvolvimento e produção de alimentos para consumo humano e animal. Rio de Janeiro: Embrapa Agroindústria de Alimentos. 2015. 100p.

AWOLU, O.O.; OLUWAFERANMI, P.M.; FAFOWORA, O. I.; OSEYEMI, G. F. Optimization of the extrusion process for production of ready-to-eat snack from rice, cassava and kersting's groundnut composite flours. LWT- Food Science and Technology, v.64, n.1, p.18-24, 2015.

BERK, Z. Food process engeneering and technology. Academic Press. New York, 2009. p.333-350.

BOX, G. E. P. Exploration of maxima and ridge systems with second-order response surfaces. In: Empirical model-building and response surfaces. Eds. G. E. P. Box John Wiley \& Sons, New York. 1987. p.304-322. 
BRAM, P.; FAISAL,T.; GREET, K.; KRISTOF, B.; HANS, G.;MARTINE, W.; JANA, D. The role of sugar and fat in sugar-snap cookies: structural and textural properties. Journal of Food Engineering, v.90, n.3, p. 400-408, 2009.

CÓRDOVA, K. R. V.; GAMA, T. M. M.T. B.; WINTER, C.M.G.; KASKANTZIS NETO G.; FREITAS, R. J. S. Características físico-químicas da casca do maracujá amarelo (Passiflora edulis flavicarpa Degener) obtida por secagem. Boletim do Centro de Pesquisa de Processamento de Alimentos. Curitiba, v.23, n.2, p.221-230, 2005.

EL-DASH, A.A.; CAMARGO, C.O.; DIAZ, N. M. Fundamentos da tecnologia de panificação. Série Tecnologia Agroindustrial. São Paulo: Fundação Tropical de Pesquisas e Tecnologia Agroindustrial, 1982. 349p.

FARO, H.C. Doença celíaca: revisão bibliográfica.2008. 95f. Monografia (Especialização em Pediatria) - Hospital Regional da Asa Sul, Brasília, 2008.

FEDDERN, V.; DURANTE, V.V.O.; MIRANDA, M.Z.; MELLADO, M. L. M. S. Avaliação física e sensorial de biscoito tipo cookie adicionados de farelo de trigo e arroz. Brazilian Journal of Food Technology, v.14, n.4, p.267-274, 2011.

FENACELBRA- Federação Nacional das Associações de Celíacos do Brasil. Guia orientador para celíacos. Ministério da Justiça. 2010.

FERREIRA, S.M.R.; LUPARELLI, P.C.; SCHIEFERDECKER, M. E.M.; VILELA, R.M. Cookies sem glúten a partir da farinha de sorgo. Archivos Latinoamericanos de Nutrición, v.59, n.4, p.433-440, 2009.

GALISTEO, M.; DUARTE, J.; ZARZUELO, A. Effects of dietary fibers on disturbances clustered in the metabolic syndrome. Journal of Nutritional Biochemistry, v.19, n.2, p. 7184, 2008.

GISSlEN, W. Panificação \& Confeitaria Profissionais. Le Cordon Bleu. Academia de Artes Culinárias de Paris. 5ed. Barueri: Manole, 2014.

GÖKMEN, V.; SERPEN, A.; AÇAR, O.C.; MORALES, F.J. Significance of furosine as heatinduced marker in cookies. Journal of Cereal Science, v.48, n.3, p.843-847, 2008.

IBGE. Instituto Brasileiro de Geografia e Estatística. Produção Agrícola Municipal, 2018.

INSTITUTE OF FOOD TECHNOLOGISTS. Sensory Evaluation Division. Sensory evaluation guide for testing food and beverage products. Food Technology., v.35, n.11, p.50$59,1981$.

INSTITUTO ADOLFO LUTZ. Normas analíticas do Instituto Adolfo Lutz. $3^{\text {a }}$ edição. São Paulo, v.1, p.533, 1985.

LA BARCA, A.M.; ROJAS-MARTÍNEZ, M.E.; ISLAS-RUBIO, A.R.; CABRERACHÁVEZ, F. Glúten-freee breads and cookies of raw and popped amaranth flours with 
attractive technological and nutritional qualities. Plant Foods for Human Nutrition, Dordrecht, v.65, n.3, p.241-246, 2010.

MARIANI, M.; OLIVEIRA, V.R.; FACCIN, R.; RIOS, A. O.; VENZKE, J.G. Elaboração e avaliação de biscoitos sem glúten a partir de farelo de arroz e farinhas de arroz e soja. Brazilian Journal of Food Technology, Campinas, v.18, n.1, p.70-78, 2015.

MELETTI, L.M.M. Avanços na cultura do maracujá no Brasil. Revista Brasileira de Fruticultura, Jaboticabal,v.33, n.spe1, p.83-91, 2011.

NABESHIMA, E. H.; EL-DASH, A. A. Modificação química da farinha de arroz como alternativa para o aproveitamento dos subprodutos do beneficiamento do arroz. Boletim do Centro de Pesquisa Processamento de Alimentos, Curitiba, v.22, n.1, p.107-120, 2004.

NASCIMENTO, N.C.; MEDEIROS, H.I.R.; PEREIRA, I.C.; OLIVEIRA, R.E.S.; MEDEIROS, I. L.; JÚNIOR, F.C.M. Elaboração do biscoito com a farinha da casca do maracujá (Passiflora edulis). Research, Society and Development, v.9, n.7, e501974333, 2020.

OLIVEIRA, D. I.; KOLAKOWSKI, A.P.; SIMÕES, D.R.S.; LOS, P.R.; DEMIATE, I.M. Biscoitos tipo cookie sem glúten formulados com farelo de feijão, farinha de arroz e amido de mandioca. Revista Brasileira de Tecnologia Agroindustrial, Ponta Grossa, v.11, n.2, p. 2502-2522, 2017.

OLIVEIRA, A.; CURTA, C. C. Cookie isento de glúten obtido com biomassa e farinha de banana (Musa paradisíaca)verde. Paraná, 2014. Dissertação de Graduação em Alimentos.

OLIVEIRA, C. A. O.; ANSELMI, A. A.; KOLLING, FINGER, M. I. F.; DALLA, CORTE, V. F.; DILL, M. D. Farinha de arroz e derivados como alternativas para a cadeia produtiva do arroz. Revista Brasileira de Produtos Agroindustriais, Campina Grande, v.16, n.3, p.291297, 2014.

PANG, Y.; AHMED, S.; XU, Y.; BETA, T.; ZHU,Z.; SHAO, Y.; BAO, J. Bound phenolic compounds and antioxidant properties of whole grain and bran of white, red and black rice. Food Chemistry, v.240, p.212-221, 2018.

RAMOS, F.S.A.R.; SANTOS, T.C.; FERREIRA,T.H.B.; GOMES, M.C.S.; MUNHOZ, C.L. Aceitabilidade de biscoito tipo cookie enriquecidos com farinha de jatobá. Cadernos de Agroecologia, v.13,n.2, 2018.

RAHAIE, S.; GHARIBZAHEDI, S. M. T.; RAZAVI, S.H.; JAFARI, S.M. Desenvolvimentos recentes sobre novas fórmulas baseadas em ingredientes nutrientes para a produção de pão saudável-funcional:uma revisão. Journal of Food Science and Tecnology, Mysore, v.51, n.11, p.2896-2906, 2014.

SANTOS, D. S. D.; STORCK, C. R.; FOGAÇA, A.O. Biscoito com adição de farinha de casca de limão. Ciência da Saúde, Santa Maria, v.15, n.1, p.123-135, 2014. 
SILVA, S.R.; PINTO, E. G.; SOARES, D. Biscoito tipo cookie de farinha de amêndoa de pequi: avaliação física e química. Enciclopédia Biosfera, Goiânia, v.15, n.27, 1401-1410, 2018.

SIVARAMAKRISHNAN, H.P.; SENGE, B.; CHATTOPADHYAY,P.K. Rheological properties of rice dough for making rice bread. Journal of Food Engineering, Essex, v.62, n.1, p.37-45, 2004.

STONE, H.; SIDEL, J. L. Sensory Evaluation Practices. Academic Press, Orlando, 1985. 310 p.

YAPO, B.M.; KOFFI, K. L. Yellow passion fruit rind - A potential source of low-methoxyl pectin. Journal of Agricultural and Food Chemistry, Whasington, v.54, n.7, p.2738-2744, 2006.

ZERAIK, M.L.; YARIWAKE, J.H. Analysis of passion fruit rinds (Passiflora edulis):isoorientin quantification by HPTLC and evaluation of antioxidant (radical scavenging) capacity. Química Nova, São Paulo, v.35, n.3, p.541-545, 2012.

ZERAIK, M. L.; PEREIRA, C. A. M.; ZUIN, V.G.; YARIWAKE,J.H. Maracujá: um alimento funcional?. Revista Brasileira de Farmacognosia, Curitiba, v.20, n.3, p.459-471, 2010 .

ZUCOLOTTO, S.M.; FAGUNDES, C.; REGINATTO, F.H.; RAMOS, F.A.; CASTELLANOS, L.; DUQUE, C.; SCHENKEL, E.P. Analysis of C-glycosyl flavonoids from South American Passiflora Species by HPLC-DAD and HPLC-MS. Phytochemical Analysis, v.23, p.232-239, 2012.

ZUCCO, F.; BORSUK, Y.; ARNTFIELD, S.D. Physical and nutritional evaluation of wheat cookies supplemented with pulse flours of different particles sizes. LWT- Food Science and Tecnology, Campinas, v.44, n.10, p.2070-2076, 2011. 